\title{
Angiotensin II type 1 receptor blockers as a first choice in patients with acute myocardial infarction
}

\author{
Jang Hoon Lee ${ }^{1}$, Myung Hwan Bae ${ }^{1}$, Dong Heon Yang ${ }^{1}$, Hun Sik Park ${ }^{1}$, Yongkeun Cho', Won Kee Lee ${ }^{2}$, \\ Myung Ho Jeong 3 , Young Jo Kim ${ }^{4}$, Myeong Chan $\mathrm{Cho}^{5}$, Chong Jin Kim ${ }^{6}$, Shung Chull Chae ${ }^{1}$; and Korea \\ Acute Myocardial Infarction Registry Investigators
}

\begin{abstract}
Departments of ${ }^{1}$ Internal Medicine and ${ }^{2}$ Preventive Medicine, Kyungpook National University School of Medicine, Daegu; ${ }^{3}$ Department of Internal Medicine, Chonnam National University Hospital, Gwangju; ${ }^{4}$ Department of Internal Medicine, Yeungnam University Medical Center, Daegu; ${ }^{5}$ Department of Internal Medicine, Chungbuk National University School of Medicine, Cheongju; ${ }^{6}$ Department of Internal Medicine, Kyung Hee University East-West Neo Medical Center, Seoul, Korea
\end{abstract}

Received: September 4, 2014 Revised : November 19, 2014 Accepted: December 17, 2014

\section{Correspondence to}

Shung Chull Chae, M.D.

Department of Internal Medicine, Kyungpook National University Hospital, 130 Dongdeok-ro, Junggu, Daegu 41944, Korea

Tel: $+82-53-420-5527$

Fax: +82-53-426-2046

E-mail: scchae@knu.ac.kr
Background/Aims: Angiotensin II type 1 receptor blockers (ARBs) have not been adequately evaluated in patients without left ventricular (LV) dysfunction or heart failure after acute myocardial infarction (AMI).

Methods: Between November 2005 and January 2008, 6,781 patients who were not receiving angiotensin-converting enzyme inhibitors (ACEIs) or ARBs were selected from the Korean AMI Registry. The primary endpoints were 12-month major adverse cardiac events (MACEs) including death and recurrent AMI.

Results: Seventy percent of the patients were Killip class 1 and had a LV ejection fraction $\geq 40 \%$. The prescription rate of ARBs was $12.2 \%$. For each patient, a propensity score, indicating the likelihood of using ARBs during hospitalization or at discharge, was calculated using a non-parsimonious multivariable logistic regression model, and was used to match the patients 1:4, yielding 715 ARB users versus 2,860 ACEI users. The effect of ARBs on in-hospital mortality and 12-month MACE occurrence was assessed using matched logistic and Cox regression models. Compared with ACEIs, ARBs significantly reduced in-hospital mortality (1.3\% vs. 3.3\%; hazard ratio [HR], 0.379 ; $95 \%$ confidence interval [CI], 0.190 to $0.756 ; p=0.006$ ) and 12-month MACE occurrence (4.6\% vs. 6.9\%; HR, 0.661; $95 \%$ CI, 0.457 to $0.956 ; p=0.028)$. However, the benefit of ARBs on 12-month mortality compared with ACEIs was marginal (4.3\% vs. $6.2 \%$; HR, $0.684 ; 95 \%$ CI, 0.467 to $1.002 ; p=0.051$ ).

Conclusions: Our results suggest that ARBs are not inferior to, and may actually be better than ACEIs in Korean patients with AMI.

Keywords: Angiotensin-converting enzyme inhibitors; Angiotensin II type 1 receptor blockers; Myocardial infarction; Mortality; Secondary prevention

\section{INTRODUCTION}

The use of angiotensin-converting enzyme inhibitors (ACEIs) is known to reduce myocardial infarction (MI) size and the risk for subsequent acute myocardial infarction (AMI), and to improve survival in post-MI patients with congestive heart failure and asymptomatic left ven- tricular (LV) systolic dysfunction [1-3]. Furthermore, the early administration of an ACEI has been shown to have additional mortality benefits in a recent clinical trial of patients with AMI [4]. According to the current guidelines, ACEIs are recommended as a standard medical therapy for secondary prevention in AMI patients, irrespective of LV function $[5,6]$. 
Angiotensin II type 1 receptor blockers (ARBs) also inhibit the renin-angiotensin system (RAS), in a manner that is different than ACEIs, via selective inhibition of the angiotensin II type 1 receptor [7]. Losartan and valsartan, both ARBs, have been proven to be "not inferior" to captopril, an ACEI, in patients with AMI and heart failure and/or LV systolic dysfunction [8-10]. However, ARBs have not been adequately compared to ACEIs in AMI patients without heart failure and/or LV dysfunction.

By using data from a large Korean Acute Myocardial Infarction Registry (KAMIR), we attempted to determine the frequency of $A R B$ prescription as the first choice in ACEI- and ARB-naive AMI patients. We describe the characteristics of the patients for whom ARBs are prescribed, and the clinical impact of prescribing ARBs instead of ACEIs as a first choice drug.

\section{METHODS}

\section{Study design and patient population}

KAMIR is a prospective open observational multicenter on-line registry of Korean AMI patients that has been maintained through the support of the Korean Society of Circulation since November 2005. Details of the KAMIR database have been previously published [11].

Between November 2005 and January 2008, 14,871 suspected AMI patients were enrolled in KAMIR at the time of admission. Baseline clinical data were available from 12,280 patients from 39 participating hospitals. Among them, 1,284 patients (10.5\%) who had already received ACEIs $(n=626)$ or ARBs $(n=720)$ before hospitalization were excluded, and 7,516 of the remaining 10,996 patients received ACEIs or ARBs during hospitalization or at discharge. Of these patients, 6,906 provided 12-month follow-up data, but 124 of these patients received both ACEIs and ARBs and were thus excluded. In total, 6,781 patients who received ACEIs $(n=5,951)$ or ARBs $(n=830)$ during hospitalization and at discharge were analyzed in this study.

AMI was diagnosed on the basis of characteristic clinical presentation, serial changes on an electrocardiogram (ECG) suggesting infarction or injury, and an increase in cardiac enzymes [12].

We analyzed baseline demographic characteristics, ini- tial presentation, initial vital signs, ECG findings, laboratory test results, procedural data, and medications. Blood samples for baseline laboratory tests, except lipid measurement, were collected at admission or before percutaneous coronary intervention. Overnight fasting blood was also tested for lipid levels. The LV ejection fraction was determined using 2-dimensional echocardiography during the index hospitalization. In-hospital complications and their management were also recorded.

The 12-month major adverse cardiac events (MACEs) were defined as death and recurrent MI. During the follow-up period, follow-up data were obtained by reviewing medical records and interviewing patients by telephone. All data were recorded on an electronic web page-based case report form.

\section{Statistical analyses}

Data are expressed as mean \pm standard deviation for continuous variables and as percentages for categorical variables. Comparisons between baseline variables were assessed with a Student $t$ test for continuous variables and a Pearson chi-square test for categorical variables. Patients were categorized into two groups: patients receiving ACEIs, and patients receiving ARBs. Since patients were not randomly assigned to ACEIs or ARBs, 1:4 propensity score (PS) matching based on their probability of using ARBs was performed to reduce the effect of treatment-selection bias and potential confounding factors in this observational study. For each patient, a PS indicating the likelihood of using ARBs during hospitalization and at discharge was calculated using a non-parsimonious multivariable logistic regression model with covariates including baseline demographic characteristics, initial presentation, initial vital signs, ECG findings, laboratory tests results, procedural data, in-hospital complications, and medications [13]. Goodness of fit of the PS was evaluated using the c-statistic and the Hosmer-Lemeshow test. In the PS-matched cohort, the risk of each outcome was compared using Cox regression models. MACE and mortality were compared by Kaplan-Meier survival curves. For all analyses, a two-sided $p<0.05$ was considered statistically significant. Statistical analysis was performed using SAS version 9.1. (SAS Institute Inc., Cary, NC, USA). 


\section{RESULTS}

Baseline characteristics and use of medications before and after PS matching are shown in Tables 1 and 2, respectively. Overall, 4,241 of 6,098 patients (70\%) in which both the LV ejection fraction and Killip status were available were Killip class 1 and had an LV ejection fraction $\geq 40 \%$. Before PS matching, $12.2 \%$ of the patients were prescribed ARBs. Patients receiving ARBs were older, thinner, and more likely to have dyspnea, preinfarct angina pectoris, higher heart rate, higher Killip classes, atrial flutter/fibrillations at admission; during hospitalization, they were more likely to have hypertension, diabetes mellitus, previous congestive heart failure, LV dysfunction, higher glucose, and elevated creatinine levels. In contrast, ACEI users were more likely to be male, current smokers, and to have typical chest pain, ST-segment elevation MI, higher creatine kinase-MB levels, and ventricular tachyarrhythmias. Defibrillation/ cardioversion were more frequently required among ACEIs users. Anti-platelet agents, except for aspirin and B-blockers, were more frequently prescribed for ACEI users, whereas diuretics were more frequently prescribed for ARB users. The GRACE (Global Registry of Acute Coronary Event) risk score was significantly higher for ARB users compared with ACEI users before PS matching (112.7 vs. 108.1, $p<0.001)$. The GRACE risk score model distinguished low-risk ( $\mathrm{n}=4,361,64.3 \%)$, intermediate-risk $(\mathrm{n}=1,873,27.6 \%)$, and high-risk $(\mathrm{n}=$
$547,8.1 \%)$ categories of patients. ARBs were used more commonly among intermediate- or high-risk patients, whereas ACEIs were used more commonly among lowrisk patients ( $p$ for trend $<0.001$ ).

After PS matching, the mean age of the 3,575 PSmatched patients was $65.7 \pm 12.3$ years old, and 2,439 (68.2\%) were men. All baseline covariates between ACEI and ARB users were balanced after PS matching. There was no significant difference in GRACE risk score between ARB and ACEIs users (111.2 vs. 110.6, $p=0.623$ ). There were no significant differences in use of ARBs or ACEIs across risk groups ( $p$ for trend $=0.718$ ).

During follow-up, 229 MACEs (6.4\%) including 209 deaths (5.8\%) and 24 recurrent MIs (0.7\%) occurred in the matched cohort (Table 3). In the logistic regression model, the in-hospital mortality was significantly lower among ARB users as compared to ACEI users (1.3\% vs. 3.3\%; odds ratio [OR], 0.379; 95\% confidence interval [CI], 0.190 to $0.756 ; p=0.006$ ). In the Cox proportional-hazards model, there were no significant differences between ARB and ACEI users in the 12-month MACE rates (3.4\% vs. 3.7\%; hazard ratio [HR], 0.911; 95\% CI, 0.584 to $1.420 ; p=0.680$ ) or mortality (3.1\% vs. 3.1\%; HR, 1.012; $95 \%$ CI, 0.633 to $1.617 ; p=0.960$ ) among hospital survivors. The 12-month MACE occurrence was significantly lower in ARB users compared with ACEI users (4.6\% vs. 6.9\%; HR, 0.661; 95\% CI, 0.457 to $0.956 ; p=0.028)$. The difference between 12-month mortality rates among ARB (4.3\%) and ACEI (6.2\%) users was marginal (HR,

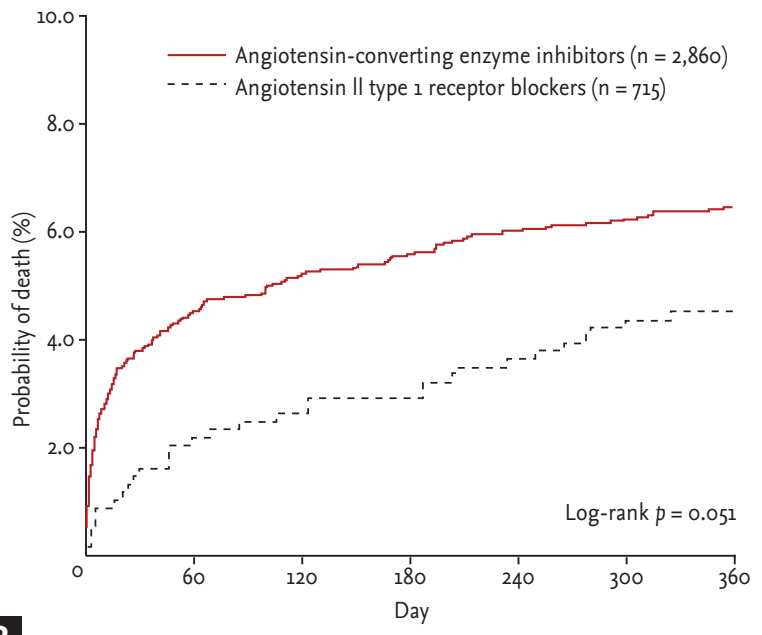

A

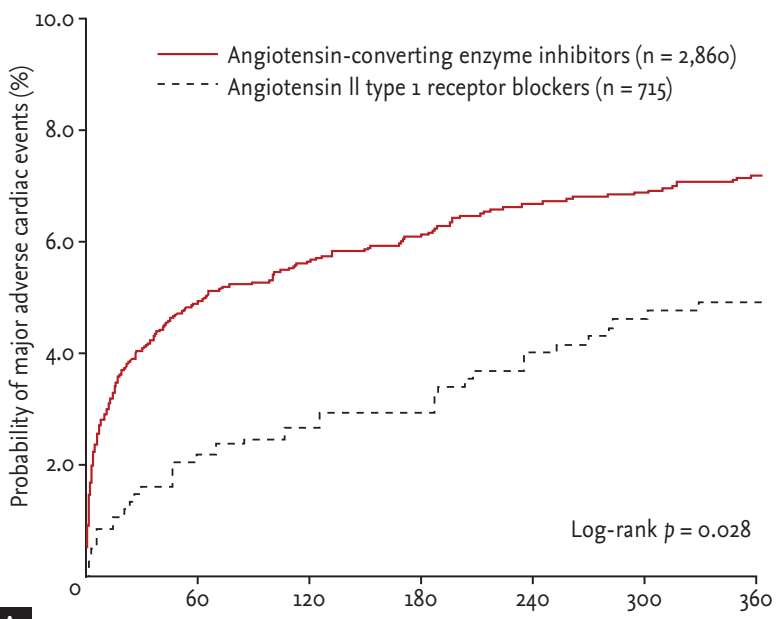

Figure 1. Kaplan-Meier estimates of the rate of 12-month major adverse cardiac events (A) and mortality (B) associated with the use of angiotensin-converting enzyme inhibitors and angiotensin II type 1 receptor blockers. 


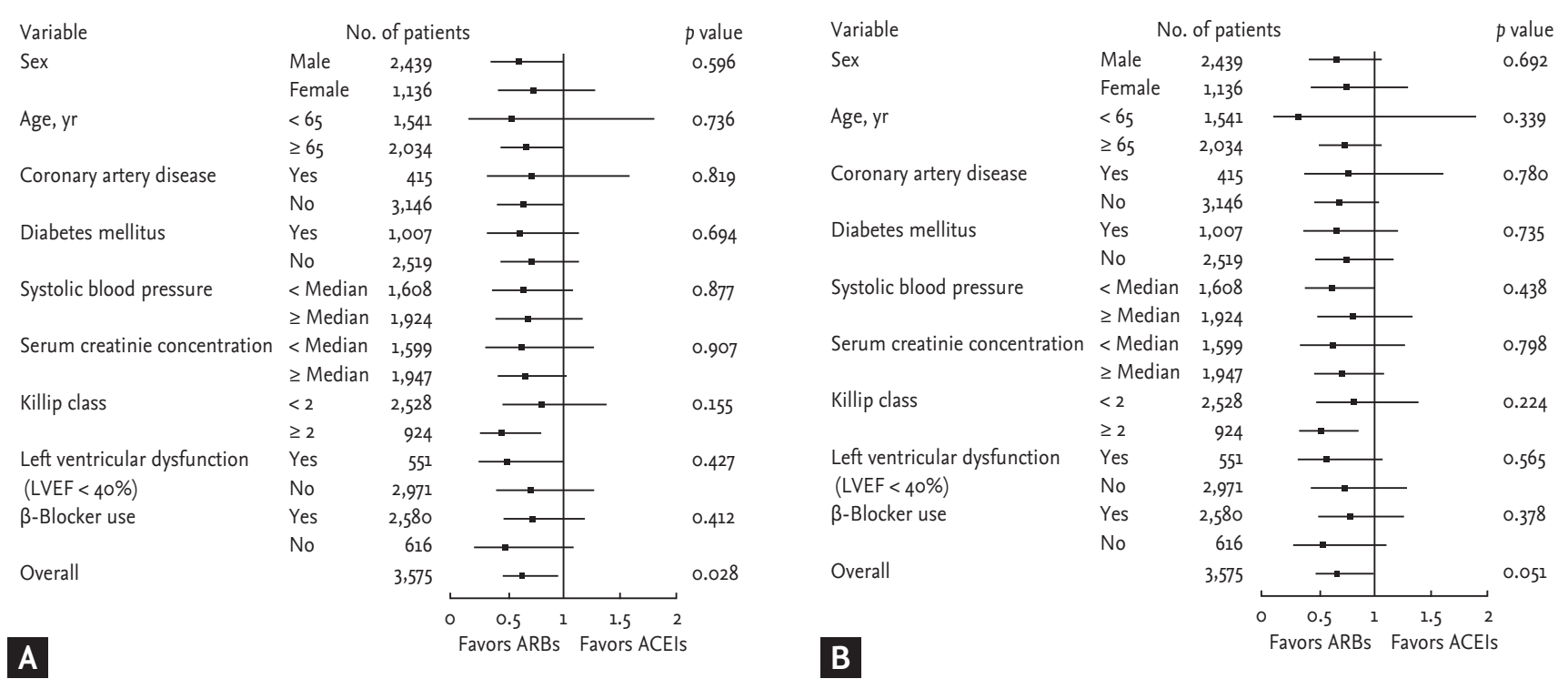

Figure 2. Hazard ratios and 95\% confidence intervals for 12-month major adverse cardiac events occurrence (A) and mortality (B). ARBs, angiotensin II type 1 receptor blockers; ACEIs, angiotensin-converting enzyme inhibitors.

$0.684 ; 95 \%$ CI, 0.467 to 1.002; $p=0.051)$. Kaplan-Meier survival estimates for 12-month MACE and mortality in the matched cohort are displayed in Fig. 1. The rate of 12-month MACE increased significantly in ACEI users subsequent to the acute phase of AMI during hospitalization. Among hospital survivors, there was no significant difference in 12-month MACE between ARB users and ACEI users. The examination of subgroups showed no heterogeneity in the effect of treatment on the risk for 12-month MACE and mortality (Fig. 2).

\section{DISCUSSION}

In this multicenter observational study, the main findings were that ARBs were commonly used as the first therapy in AMI patients, particularly in those with highrisk features, and that ARB users had significantly lower in-hospital mortality and 12-month MACEs compared with ACEI users after PS matching.

The ARBs, theoretically, might be more efficacious than ACEIs at blocking RAS. In AMI patients with heart failure and/or LV dysfunction, the efficacy of ARBs compared to ACEIs for secondary prevention of MACEs has been tested in two large-scale randomized clinical studies (RCTs): OPTIMAAL (Optimal Trial in Myocar- dial Infarction with Angiotensin II antagonist Losartan) and VALIANT (Valsartan in Acute Myocardial Infarction Trial) $[8,10]$. In OPTIMAAL, losartan was associated with a significant increase in cardiovascular mortality compared to captopril. In VALIANT, valsartan was "non-inferior" to captopril in patients with high risk for MACE after AMI. Neither of these studies revealed any statistically significant difference in the primary end-points between patients treated with ACEIs and those treated with ARBs. Therefore, ACEIs are recommended as the first choice for AMI patients with heart failure and/or LV dysfunction in the current guidelines, assigning ARBs as an alternative for ACEI-intolerant patients [5,6]. However, ARBs are commonly prescribed instead of ACEIs in post-MI patients, as shown in the REACH (Reduction of Atherothrombosis for Continued Health) registry [14]. Interestingly, ARBs were also used in our observational study as the first choice RAS blockade in AMI patients, particularly in patients with high-risk features.

In the present study, ARBs significantly reduced in-hospital mortality and 12-month MACE occurrence compared with ACEIs. The rate of 12-month MACE occurrence diverged during the very acute phase of AMI during hospitalization, a period of drug dose increase, with no further subsequent divergence. Although the better prognosis among ARB users may have been the result of chance, 
Table 1. Baseline characteristics associated with the use of angiotensin-converting enzyme inhibitors or angiotensin II type 1 receptor blockers before and after propensity score matching

\begin{tabular}{|c|c|c|c|c|c|c|}
\hline \multirow[b]{2}{*}{ Characteristic } & \multicolumn{3}{|c|}{ Before PS match } & \multicolumn{3}{|c|}{ After PS match } \\
\hline & $\begin{array}{l}\text { ACEIs alone } \\
(\mathrm{n}=5,951)\end{array}$ & $\begin{array}{l}\text { ARBs alone } \\
(\mathrm{n}=830)\end{array}$ & $p$ value & $\begin{array}{l}\text { ACEIs alone } \\
(\mathrm{n}=2,860)\end{array}$ & $\begin{array}{c}\text { ARBs alone } \\
(\mathrm{n}=715)\end{array}$ & $p$ value \\
\hline \multicolumn{7}{|l|}{ Demographic } \\
\hline Age, yr & $64.5 \pm 12.6$ & $66.2 \pm 12.3$ & $<0.001$ & $65.7 \pm 12.3$ & $65.8 \pm 12.3$ & 0.855 \\
\hline Male sex & $4,357(73.2)$ & $543(65 \cdot 4)$ & $<0.001$ & $1,951(68.2)$ & $488(68.3)$ & 0.986 \\
\hline Height, cm & $163.9 \pm 8.7$ & $162.7 \pm 9.3$ & 0.001 & $163.0 \pm 8.8$ & $163.0 \pm 9.2$ & 0.948 \\
\hline Weight, kg & $64.7 \pm 11.4$ & $63.6 \pm 11.4$ & 0.008 & $63.9 \pm 11.5$ & $63.8 \pm 11.3$ & 0.933 \\
\hline Body mass index, $\mathrm{kg} / \mathrm{m}^{2}$ & $24.0 \pm 3 \cdot 3$ & $23.9 \pm 3.1$ & 0.383 & $23.9 \pm 3.2$ & $23.9 \pm 3.1$ & 0.979 \\
\hline \multicolumn{7}{|l|}{ Initial presentation } \\
\hline Pre-hospital resuscitation & $96(1.6)$ & $15(1.8)$ & 0.680 & $48(1.7)$ & $14(2.0)$ & 0.607 \\
\hline Typical chest pain & $4,976(85.0)$ & $664(81.2)$ & 0.005 & $2,332(82.8)$ & $588(83.2)$ & 0.822 \\
\hline Dyspnea & $1,344(23.7)$ & $225(28.1)$ & 0.006 & $695(25 \cdot 4)$ & $183(26.5)$ & 0.547 \\
\hline Pre-infarction angina pectoris & $2,540(43 \cdot 3)$ & $395(47 \cdot 7)$ & 0.017 & $1,296(45.8)$ & $329(46.1)$ & 0.880 \\
\hline Systolic blood pressure, $\mathrm{mmHg}$ & $129.8 \pm 28.1$ & $130.8 \pm 29.9$ & 0.355 & $130.9 \pm 29.0$ & $131.0 \pm 29.4$ & 0.932 \\
\hline Heart rate, beats/min & $77 \cdot 3 \pm 19 \cdot 3$ & $79.1 \pm 20.5$ & 0.016 & $78.1 \pm 19.5$ & $78.5 \pm 19.8$ & 0.639 \\
\hline Killip class $>1$ & $1,265(22.2)$ & $251(30.9)$ & $<0.001$ & $722(26.2)$ & $202(29.0)$ & 0.132 \\
\hline \multicolumn{7}{|l|}{ ECG on admission } \\
\hline ST elevation myocardial infarction & $3,700(62.2)$ & $485(58.4)$ & 0.038 & $1,750(61.2)$ & $435(60.8)$ & 0.864 \\
\hline Anterior myocardial infarction & $2,642(46.8)$ & $383(48.2)$ & 0.462 & $1,326(48.4)$ & $328(47 \cdot 9)$ & 0.817 \\
\hline Inferior myocardial infarction & $2,043(36.2)$ & $299(37.6)$ & 0.432 & $1,009(36.8)$ & $255(37.2)$ & 0.840 \\
\hline \multicolumn{7}{|l|}{ Heart rhythm on admission } \\
\hline Sinus rhythm & $5,270(91.6)$ & $737(90.4)$ & 0.275 & $2,557(91.7)$ & $634(90.6)$ & 0.318 \\
\hline Atrial flutter/fibrillation & $223(3.9)$ & $46(5 \cdot 6)$ & 0.017 & $128(4.6)$ & $37(5 \cdot 3)$ & 0.440 \\
\hline $\begin{array}{l}\text { Ventricular tachycardia/ } \\
\text { ventricular fibrillation }\end{array}$ & $28(0.5)$ & $3(0.4)$ & 0.644 & $11(0.4)$ & $3(0.4)$ & 0.899 \\
\hline \multicolumn{7}{|l|}{ Past history } \\
\hline Previous coronary heart disease & $660(11.1)$ & $104(12.6)$ & 0.229 & $330(11.6)$ & $85(11.9)$ & 0.803 \\
\hline Hypertension $^{\mathrm{a}}$ & $2,587(44.0)$ & $447(54 \cdot 4)$ & $<0.001$ & $1,481(52 \cdot 3)$ & $366(51.8)$ & 0.795 \\
\hline Diabetes mellitus $^{\mathrm{b}}$ & $1,495(25.5)$ & $256(31.3)$ & $<0.001$ & $801(28.4)$ & $206(29 \cdot 3)$ & 0.626 \\
\hline Hyperlipidemia $^{c}$ & $536(10.2)$ & $71(9.8)$ & 0.728 & $233(9.2)$ & $60(9.6)$ & 0.763 \\
\hline Current smoking & $2,792(47 \cdot 3)$ & $316(38.3)$ & $<0.001$ & $1,152(40.6)$ & $292(41.1)$ & 0.801 \\
\hline Previous congestive heart failure & $77(1.3)$ & $18(2.2)$ & 0.045 & $40(1.4)$ & $13(1.8)$ & 0.406 \\
\hline Previous cerebrovascular disease & $358(6.0)$ & $52(6.3)$ & 0.778 & $173(6.0)$ & $47(6.6)$ & 0.602 \\
\hline Left ventricular ejection fraction & $52.0 \pm 13.0$ & $50.9 \pm 13.4$ & 0.023 & $51.3 \pm 12.6$ & $51.1 \pm 13.1$ & 0.754 \\
\hline \multicolumn{7}{|l|}{ Laboratory findings } \\
\hline Glucose, mg/dL & $167.0 \pm 76.4$ & $174.2 \pm 87.4$ & 0.026 & $170.2 \pm 80.5$ & $172.6 \pm 80.8$ & 0.489 \\
\hline Creatinine, mg/dL & $1.09 \pm 0.94$ & $1.34 \pm 1.83$ & $<0.001$ & $1.11 \pm 0.91$ & $1.18 \pm 1.13$ & 0.142 \\
\hline CK-MB, ng/mL & $142.6 \pm 241.4$ & $122.6 \pm 179.1$ & 0.004 & $129 \cdot 3 \pm 169.4$ & $129.1 \pm 186.0$ & 0.976 \\
\hline Total cholesterol, mg/dL & $185.0 \pm 44.0$ & $186.3 \pm 47.9$ & 0.451 & $187 \cdot 3 \pm 45 \cdot 4$ & $186.3 \pm 45 \cdot 3$ & 0.608 \\
\hline Triglyceride, mg/dL & $129.2 \pm 109.0$ & $129.6 \pm 82.4$ & 0.929 & $133.0 \pm 128.0$ & $129.1 \pm 80.4$ & 0.448 \\
\hline HDL-C, mg/dL & $45.6 \pm 20.9$ & $44.8 \pm 22.0$ & 0.381 & $45.2 \pm 14.1$ & $45.2 \pm 23.1$ & 0.962 \\
\hline
\end{tabular}


Table 1. Continued

\begin{tabular}{|c|c|c|c|c|c|c|}
\hline \multirow[b]{2}{*}{ Characteristic } & \multicolumn{3}{|c|}{ Before PS match } & \multicolumn{3}{|c|}{ After PS match } \\
\hline & $\begin{array}{l}\text { ACEIs alone } \\
(\mathrm{n}=5,951)\end{array}$ & $\begin{array}{l}\text { ARBs alone } \\
(\mathrm{n}=830)\end{array}$ & $p$ value & $\begin{array}{c}\text { ACEIs alone } \\
(\mathrm{n}=2,860)\end{array}$ & $\begin{array}{l}\text { ARBs alone } \\
(\mathrm{n}=715)\end{array}$ & $p$ value \\
\hline $\mathrm{LDL}-\mathrm{C}, \mathrm{mg} / \mathrm{dL}$ & $119.4 \pm 40.2$ & $119.9 \pm 43.4$ & 0.726 & $120.5 \pm 41.1$ & $120.4 \pm 43.4$ & 0.952 \\
\hline PCI at index hospitalization & $5,090(85.5)$ & $711(85 \cdot 7)$ & 0.920 & $2,474(86.5)$ & $617(86.3)$ & 0.883 \\
\hline CABG at index hospitalization & $84(1.4)$ & $14(1.7)$ & 0.518 & $44(1.5)$ & $10(1.4)$ & 0.775 \\
\hline \multicolumn{7}{|l|}{ In-hospital complication } \\
\hline Cardiogenic shock & $379(6.4)$ & $51(6.1)$ & 0.804 & $168(5 \cdot 9)$ & $42(5 \cdot 9)$ & 1.000 \\
\hline New atrial flutter/fibrillation & $43(0.7)$ & $14(1.7)$ & 0.004 & $28(1.0)$ & $6(0.8)$ & 0.730 \\
\hline $\begin{array}{l}\text { Ventricular tachycardia/ventricular } \\
\text { fibrillation during hospitalization }\end{array}$ & $235(3.9)$ & $16(1.9)$ & 0.004 & $63(2.2)$ & $16(2.2)$ & 0.955 \\
\hline Heart failure & $1,059(17.8)$ & $170(20.5)$ & 0.060 & $555(19 \cdot 4)$ & $140(19.6)$ & 0.916 \\
\hline Acute renal failure & $30(0.5)$ & $4(0.5)$ & 0.932 & $12(0.4)$ & $3(0.4)$ & 1.000 \\
\hline Cerebrovascular accidents & $46(0.8)$ & $4(0.5)$ & 0.359 & $14(0.5)$ & $4(0.6)$ & 0.813 \\
\hline Major bleeding & $14(0.2)$ & $4(0.5)$ & 0.196 & $10(0.3)$ & $2(0.3)$ & 0.772 \\
\hline \multicolumn{7}{|l|}{$\begin{array}{l}\text { Management of in-hospital } \\
\text { complication }\end{array}$} \\
\hline In-hospital resuscitation & $160(2.7)$ & $17(2.0)$ & 0.278 & $58(2.0)$ & $14(2.0)$ & 0.905 \\
\hline Defibrillation/cardioversion & $164(2.8)$ & $11(1.3)$ & 0.015 & $44(1.5)$ & $11(1.5)$ & 1.000 \\
\hline Mechanical ventilator & $217(3.6)$ & $27(3 \cdot 3)$ & 0.569 & $86(3.0)$ & $22(3.1)$ & 0.922 \\
\hline
\end{tabular}

Values are presented as mean $\pm \mathrm{SD}$ or number (\%).

PS, propensity score; ACEIs, angiotensin-converting enzyme inhibitors; ARBs, angiotensin II type 1 receptor blockers; ECG, electrocardiogram; CK-MB, creatine kinase-MB; HDL-C, high-density lipoprotein cholesterol; LDL-C, low-density lipoprotein cholesterol; PCI, percutaneous coronary intervention; CABG, coronary artery bypass grafting.

${ }^{a}$ Defined as previously diagnosed by a physician, receiving medication to lower blood pressure.

${ }^{\mathrm{b}}$ Defined as previously diagnosed by a physician, receiving medication to lower blood glucose.

${ }^{\mathrm{c}}$ Defined as previously diagnosed by a physician, receiving lipid lowering drugs.

this study suggests that it would be worth conducting RCTs to compare ARBs and ACEIs as first choice RAS blockades in AMI patients without heart failure and/or LV dysfunction.

Our study differs from the previous RCTs in several aspects. First, KAMIR was an observational study including AMI patients irrespective of the presence of heart failure and/or LV systolic dysfunction. In clinical trials on the comparative effects of RAS blockers, AMI patients without heart failure and/or LV dysfunction were not included in sizable numbers. In the present analysis, $70 \%$ of patients did not have heart failure and/or LV systolic dysfunction. Second, dosages of ACEIs and ARBs used in observational studies like ours tend to be lower compared with those in RCTs. Recent clinical studies reported that the average dosages of ACEIs used after discharge were $50 \%$ of the effective dose determined by
RCTs, with no significant differences in MACEs between ARBs and ACEIs at the dosages used [15]. Therefore, the discrepancy in clinical outcomes between previous RCTs and our registry might be due to dosage differences. In this regard, registry data better reflect the clinical efficacy of ACEIs and ARBs in real-world practice. Third, methodological differences may have contributed to observed differences. In the present study, we excluded patients who had already received ACEIs or ARBs before hospitalization, whereas in VALIANT, $39.4 \%$ of the valsartan group and $38.5 \%$ of the captopril group received non-study ACEIs for an average of 5 days after the AMI, but before randomization [10]. ACEIs are known to reduce mortality in the early post-MI period, with $85 \%$ of the benefit in the first week [16]. Therefore, the early use of non-study ACEIs in VALIANT may have influenced the results. The mortality rates of ACEI-naive patients 
Table 2. Medications prescribed in conjunction with angiotensin-converting enzyme inhibitors or angiotensin II type 1 receptor blockers before and after propensity score matching

\begin{tabular}{|c|c|c|c|c|c|c|}
\hline \multirow[b]{2}{*}{ Variable } & \multicolumn{3}{|c|}{ Before PS match } & \multicolumn{3}{|c|}{ After PS match } \\
\hline & $\begin{array}{l}\text { ACEIs alone } \\
(\mathrm{n}=5,951)\end{array}$ & $\begin{array}{c}\text { ARBs alone } \\
(\mathrm{n}=830)\end{array}$ & $p$ value & $\begin{array}{l}\text { ACEIs alone } \\
(\mathrm{n}=2,860)\end{array}$ & $\begin{array}{c}\text { ARBs alone } \\
(\mathrm{n}=715)\end{array}$ & $p$ value \\
\hline \multicolumn{7}{|l|}{ Previous medication } \\
\hline Aspirin & $357(6.0)$ & $85(10.2)$ & $<0.001$ & $231(8.1)$ & $62(8.7)$ & 0.604 \\
\hline Clopidogrel & $95(1.6)$ & $25(3.0)$ & 0.004 & $57(2.0)$ & $18(2.5)$ & 0.381 \\
\hline$\beta$-Blocker & $261(4 \cdot 4)$ & $51(6.1)$ & 0.023 & $146(5 \cdot 1)$ & $40(5 \cdot 6)$ & 0.598 \\
\hline Lipid-lowering drug & $170(2.9)$ & $37(4 \cdot 5)$ & 0.012 & $102(3.6)$ & $25(3 \cdot 5)$ & 0.928 \\
\hline Calcium channel blocker & $399(6.7)$ & $89(10.7)$ & $<0.001$ & $236(8.3)$ & $5^{8}(8.1)$ & 0.903 \\
\hline Diuretic & $152(2.6)$ & $30(3 \cdot 6)$ & 0.077 & $92(3.2)$ & $23(3.2)$ & 1.000 \\
\hline Nitrate & $158(2.7)$ & $30(3.6)$ & 0.115 & $81(2.8)$ & $23(3.2)$ & 0.584 \\
\hline \multicolumn{7}{|l|}{ In-hospital medications } \\
\hline Aspirin & $5,908(99 \cdot 3)$ & $823(99.2)$ & 0.703 & $2,836(99.2)$ & $709(99.2)$ & 1.000 \\
\hline Clopidogrel & $5,837(98.1)$ & $800(96.4)$ & 0.001 & $2,787(97 \cdot 4)$ & $694(97 \cdot 1)$ & 0.565 \\
\hline$\beta$-Blocker & $5,072(85.2)$ & $596(71.8)$ & $<0.001$ & $2,252(78.7)$ & $560(78.3)$ & 0.807 \\
\hline Lipid-lowering drug & $4,839(81.3)$ & $662(79.8)$ & 0.283 & $2,315(80.9)$ & $583(81.5)$ & 0.717 \\
\hline Heparin & $4,795(80.6)$ & $671(80.8)$ & 0.854 & $2,317(81.0)$ & $580(81.1)$ & 0.949 \\
\hline Calcium channel blocker & $619(10.4)$ & $182(21.9)$ & $<0.001$ & $394(13.8)$ & $119(16.6)$ & 0.056 \\
\hline Diuretic & $1,697(28.5)$ & $344(41.4)$ & $<0.001$ & $1,072(37.5)$ & $273(38.2)$ & 0.730 \\
\hline Nitrate & $4,846(81.4)$ & $667(80.4)$ & 0.459 & $2,307(80.7)$ & $579(81.0)$ & 0.849 \\
\hline \multicolumn{7}{|l|}{ Discharge medication } \\
\hline Aspirin & $5,578(98.2)$ & $787(97.3)$ & 0.066 & $2,684(98.0)$ & $683(97.6)$ & 0.488 \\
\hline Clopidogrel & $5,343(94.1)$ & $732(90.5)$ & $<0.001$ & $2,554(93.2)$ & $643(91.9)$ & 0.200 \\
\hline$\beta$-Blocker & $4,597(80.9)$ & $557(68.9)$ & $<0.001$ & $2,044(74 \cdot 6)$ & $515(73 \cdot 6)$ & 0.568 \\
\hline Lipid-lowering drug & $4,570(80.5)$ & $641(79.2)$ & 0.407 & $2,202(80.4)$ & $560(80.0)$ & 0.815 \\
\hline Calcium channel blocker & $428(7.5)$ & $148(18.3)$ & $<0.001$ & $308(11.2)$ & $79(11.3)$ & 0.976 \\
\hline Diuretic & $1,136(20.0)$ & $264(32.6)$ & $<0.001$ & $771(28.1)$ & $206(29.4)$ & 0.503 \\
\hline Nitrate & $3,486(61.4)$ & $490(60.6)$ & 0.656 & $1,690(61.7)$ & $430(61.4)$ & 0.895 \\
\hline
\end{tabular}

Values are presented as number (\%).

PS, propensity score; ACEIs, angiotensin-converting enzyme inhibitors; ARBs, angiotensin II type 1 receptor blockers.

compared with those who received pre-randomization ACEIs in VALIANT have not yet been published [9,10]. Finally, ethnic differences may also have contributed to the observed differences. The prescription rate of ARBs is lower in Western countries than it is in Asia [14,17]. East Asians are more susceptible to the adverse side effect of coughing caused by ACEIs [18,19], which may have led to a relatively rapid switch from ACEIs to ARBs in East Asia. However, whether the drug response differences are solely the result of genetic differences between Asians and Westerners is still unknown. Furthermore, it is not known whether these differences cause different clinical outcomes for Asian post-MI patients.

Our study has several potential limitations. First, KAMIR was an observational study as previously explained. Although propensity analysis was performed for a large number of confounding factors, no firm conclusion can be made about the causal relationship between ACEIs or ABRs and clinical outcomes. Second, we were unable to clarify the reasons for nonprescription of ACEIs or ARBs. In addition, the details regarding dosages and specific class of ACEIs or ARBs and the timing of use of 
Table 3. Twelve-month clinical outcomes in the matched cohort

\begin{tabular}{|c|c|c|c|c|c|c|}
\hline \multirow{2}{*}{ End point } & \multirow{2}{*}{$\begin{array}{l}\text { ACEIs group } \\
(\mathrm{n}=2,860)\end{array}$} & \multirow{2}{*}{$\begin{array}{l}\text { ARBs group } \\
\quad(\mathrm{n}=715)\end{array}$} & \multicolumn{2}{|c|}{ ARBs vs. ACEIs } & \multicolumn{2}{|c|}{ ARBs vs. ACEIs } \\
\hline & & & OR $(95 \% \mathrm{CI})$ & $p$ value & $\operatorname{HR}(95 \% \mathrm{CI})$ & $p$ value \\
\hline In-hospital & 2,860 & 715 & & & & \\
\hline Death from any cause & $93(3 \cdot 3)$ & $9(1.3)$ & $0.379(0.190-0.756)$ & 0.006 & & \\
\hline Death from cardiac cause & $75(2.6)$ & $8(1.1)$ & $0.420(0.202-0.875)$ & 0.021 & & \\
\hline Death from non-cardiac cause & $18(0.6)$ & $1(0.1)$ & $0.221(0.029-1.659)$ & 0.142 & & \\
\hline Hospital survivors & 2,767 & 706 & & & & \\
\hline Death or recurrent MI & $103(3.7)$ & $24(3 \cdot 4)$ & & & $0.911(0.584-1.420)$ & 0.680 \\
\hline Death from any cause & $85(3.1)$ & $22(3.1)$ & & & $1.012(0.633-1.617)$ & 0.960 \\
\hline Death from cardiac cause & $61(2.2)$ & $14(2.0)$ & & & $0.897(0.502-1.604)$ & 0.715 \\
\hline Death from non-cardiac cause & $24(0.9)$ & $8(1.1)$ & & & $1.303(0.585-2.901)$ & 0.517 \\
\hline Recurrent MI & $22(0.8)$ & $2(0.3)$ & & & $0.355(0.083-1.509)$ & 0.161 \\
\hline Overall & 2,860 & 715 & & & & \\
\hline Death or recurrent MI & $196(6.9)$ & $33(4.6)$ & & & $0.661(0.457-0.956)$ & 0.028 \\
\hline Death from any cause & $178(6.2)$ & $31(4 \cdot 3)$ & & & $0.684(0.467-1.002)$ & 0.051 \\
\hline Death from cardiac cause & $136(4.8)$ & $22(3.1)$ & & & $0.636(0.406-0.998)$ & 0.049 \\
\hline Death from non-cardiac cause & $42(1.5)$ & $9(1.3)$ & & & $0.840(0.409-1.725)$ & 0.634 \\
\hline Recurrent MI & $22(0.8)$ & $2(0.3)$ & & & $0.355(0.084-1.510)$ & 0.161 \\
\hline
\end{tabular}

Values are presented as number (\%).

ACEI, angiotensin-converting enzyme inhibitor; ARB, angiotensin II type 1 receptor blocker; OR, odds ratio; CI, confidence interval; HR, hazard ratio; MI, myocardial infarction.

ACEIs or ARBs after the onset of MI were not obtained from the patients or their physicians. Finally, patients who had previously experienced adverse reactions to ACEIs or ARBs might have been included in our study. Considering this, we may have underestimated the prescription rates for ACEIs or ARBs. Finally, no data were available on compliance or adherence regarding ACEIs or ARBs throughout the follow-up period. We were unable to detect how much crossover occurred between ACEI-users and ARB-users.

In conclusion, ARBs significantly reduced in-hospital mortality and 12-month MACEs compared with ACEIs in Korean AMI patients. Further RCTs are required for AMI patients without heart failure and/or LV dysfunction.

\section{KEY MESSAGE}

1. Angiotensin II type 1 receptor blockers (ARBs) were commonly used in Korean acute myocardial infarction patients, particularly those with high-risk features, as a first choice therapy.

2. Korean ARB users had significantly lower in-hospital mortality and 12-month major adverse cardiac event occurrence compared with angiotensin-converting enzyme inhibitor users after propensity score matching.

\section{Conflict of interest}

No potential conflict of interest relevant to this article was reported.

\section{Acknowledgments}

Korean Acute Myocardial Infarction Registry Investigators: Bon-Kwon Koo, Byung-Ok Kim, Chong-Jin Kim, Chong-Yun Rhim, Dong-Hoon Choi, Dong-Kyu Jin, Doo-Il Kim, Hyeon-Cheol Gwon, In-Ho Chae, InWhan Seong, Jae-Young Rhew, Jang-Ho Bae, Jei-Keon Chae, Jeong-Gwan Cho, Jin-Man Cho, Jin-Yong Hwang, Jong-Hyun Kim, Jong-Seon Park, Jung-Han Yoon, JuYoung Yang, Kee-Sik Kim, Keum-Soo Park, Ki-Bae 
Seung, Kyoo-Rok Han, Kyoung-Tae Jeong, Moo-Hyun Kim, Myeong-Chan Cho, Myung-Ho Jeong, MyoungYong Lee, Nae-Hee Lee, Seok-Kyu Oh, Seong-Wook Park, Seong-Woon Rha, Seung-Ho Hur, Seung-Jae Joo, Seung-Jea Tahk, Seung-Jung Park, Shung-Chull Chae, Soo-Joong Kim, Tae-Hoon Ahn, Taek-Jong Hong, Yang-Soo Jang, Young-Jo Kim, Young-Keun Ahn, Young-Youp Koh, and Wook-Sung Chung.

\section{REFERENCES}

1. The CONSENSUS Trial Study Group. Effects of enalapril on mortality in severe congestive heart failure: results of the Cooperative North Scandinavian Enalapril Survival Study (CONSENSUS). N Engl J Med 1987;316:1429-1435.

2. The Acute Infarction Ramipril Efficacy (AIRE) Study Investigators. Effect of ramipril on mortality and morbidity of survivors of acute myocardial infarction with clinical evidence of heart failure. Lancet 1993;342:821-828.

3. Pfeffer MA, Braunwald E, Moye LA, et al. Effect of captopril on mortality and morbidity in patients with left ventricular dysfunction after myocardial infarction: results of the survival and ventricular enlargement trial. The SAVE Investigators. N Engl J Med 1992;327:669-677.

4. ISIS-4 (Fourth International Study of Infarct Survival) Collaborative Group. ISIS-4: a randomised factorial trial assessing early oral captopril, oral mononitrate, and intravenous magnesium sulphate in 58,050 patients with suspected acute myocardial infarction. Lancet 1995;345:669-685.

5. Van de Werf F, Bax J, Betriu A, et al. Management of acute myocardial infarction in patients presenting with persistent ST-segment elevation: the Task Force on the Management of ST-Segment Elevation Acute Myocardial Infarction of the European Society of Cardiology. Eur Heart J 2008;29:2909-2945.

6. Task Force for Diagnosis and Treatment of Non-STSegment Elevation Acute Coronary Syndromes of European Society of Cardiology, Bassand JP, Hamm CW, et al. Guidelines for the diagnosis and treatment of non-STsegment elevation acute coronary syndromes. Eur Heart J 2007;28:1598-1660.

7. Levy BI. Can angiotensin II type 2 receptors have deleterious effects in cardiovascular disease? Implications for therapeutic blockade of the renin-angiotensin system.
Circulation 2004;109:8-13.

8. Dickstein K, Kjekshus J; OPTIMAAL Steering Committee of the OPTIMAAL Study Group. Effects of losartan and captopril on mortality and morbidity in high-risk patients after acute myocardial infarction: the OPTIMAAL randomised trial. Optimal Trial in Myocardial Infarction with Angiotensin II Antagonist Losartan. Lancet 2002;360:752-760.

9. Pfeffer MA, McMurray JJ, Velazquez EJ, et al. Valsartan, captopril, or both in myocardial infarction complicated by heart failure, left ventricular dysfunction, or both. $\mathrm{N}$ Engl J Med 2003;349:1893-1906.

10. McMurray J, Solomon S, Pieper K, et al. The effect of valsartan, captopril, or both on atherosclerotic events after acute myocardial infarction: an analysis of the Valsartan in Acute Myocardial Infarction Trial (VALIANT). J Am Coll Cardiol 2006;47:726-733.

11. Lee JH, Park HS, Chae SC, et al. Predictors of six-month major adverse cardiac events in 30-day survivors after acute myocardial infarction (from the Korea Acute Myocardial Infarction Registry). Am J Cardiol 2009;104:182189.

12. Myocardial infarction redefined: a consensus document of The Joint European Society of Cardiology/American College of Cardiology Committee for the redefinition of myocardial infarction. Eur Heart J 2000;21:1502-1513.

13. Rosenbaum PR, Rubin DB. The central role of the propensity score in observational studies for causal effects. Biometrika 1983;70:41-55.

14. Bhatt DL, Steg PG, Ohman EM, et al. International prevalence, recognition, and treatment of cardiovascular risk factors in outpatients with atherothrombosis. JAMA 2006;295:180-189.

15. Hansen ML, Gislason GH, Kober L, et al. Different angiotensin-converting enzyme inhibitors have similar clinical efficacy after myocardial infarction. Br J Clin Pharmacol 2008;65:217-223.

16. ACE Inhibitor Myocardial Infarction Collaborative Group. Indications for ACE inhibitors in the early treatment of acute myocardial infarction: systematic overview of individual data from 100,000 patients in randomized trials. Circulation 1998;97:2202-2212.

17. Kohro T, Hayashi D, Okada Y, Yamazaki T, Nagai R; JCAD Investigators. Effects of medication on cardiovascular events in the Japanese coronary artery disease (JCAD) study. Circ J 2007;71:1835-1840. 
18. Morimoto T, Gandhi TK, Fiskio JM, et al. An evaluation of risk factors for adverse drug events associated with angiotensin-converting enzyme inhibitors. J Eval Clin Pract 2004;10:499-509.
19. Woo KS, Norris RM, Nicholls G. Racial difference in incidence of cough with angiotensin-converting enzyme inhibitors (a tale of two cities). Am J Cardiol 1995;75:967968. 